Existence, uniqueness, and convergence of the regularized primal-dual central path

\author{
Jordi Castro \\ Jordi Cuesta \\ Dept. of Stat. and Operations Research Dept. of Chemical Engineering \\ Universitat Politècnica de Catalunya Universitat Rovira i Virgili \\ jordi.castro@upc.edu jordi.cuesta@urv.cat \\ Research Report UPC-DEIO DR 2009-08 \\ July 2009; updated March 2010, June 2010
}

Report available from http://www-eio.upc.es/ j castro 



\title{
Existence, uniqueness, and convergence of the regularized primal-dual central path
}

\author{
Jordi Castro*,a, Jordi Cuesta ${ }^{\mathrm{b}}$ \\ ${ }^{a}$ Dept. of Statistics and Operations Research, Universitat Politècnica de Catalunya, Barcelona, Catalonia, Spain \\ ${ }^{b}$ Operations Research unit, Dept. of Chemical Engineering, Universitat Rovira i Virgili, Tarragona, Catalonia, Spain
}

\begin{abstract}
In a recent work [3] the authors improved one of the most efficient interior-point approaches for some classes of block-angular problems. This was achieved by adding a quadratic regularization to the logarithmic barrier. This regularized barrier was shown to be self-concordant, thus fitting the general structural optimization interior-point framework. In practice, however, most codes implement primal-dual path-following algorithms. This short paper shows that the primal-dual regularized central path is well defined, i.e., it exists, it is unique, and it converges to a strictly complementary primal-dual solution.
\end{abstract}

Key words:

interior-point methods, primal-dual central path, path-following methods, regularizations

\section{Introduction}

Let us consider the linear programming problem

$$
\begin{aligned}
\min & c^{T} x \\
\text { s. to } & A x=b \\
& 0 \leq x \leq u,
\end{aligned}
$$

where $x, c, u \in \mathbb{R}^{n}, b \in \mathbb{R}^{m}$, and $A \in \mathbb{R}^{m \times n}$. Note that any bounded problem can be formulated as (1). The standard logarithmic barrier problem, used in interior-point methods, associated to (1) is

$$
\begin{array}{ll}
\min & B(x, \mu) \triangleq c^{T} x+\mu\left(-\sum_{i=1}^{n} \ln x_{i}-\sum_{i=1}^{n} \ln \left(u_{i}-x_{i}\right)\right) \\
\text { s. to } & A x=b, 0<x<u,
\end{array}
$$

$\mu$ being the barrier parameter. Previously used regularized variants replaced $B(x, \mu)$ by

$$
\begin{aligned}
B_{P}(x, \mu) & \triangleq c^{T} x+\frac{1}{2}(x-\bar{x})^{T} Q_{P}(x-\bar{x}) \\
& +\mu\left(-\sum_{i=1}^{n} \ln x_{i}-\sum_{i=1}^{n} \ln \left(u_{i}-x_{i}\right)\right),
\end{aligned}
$$

${ }^{*}$ Corresponding address: Dept. of Statistics and Operations Research, Universitat Politècnica de Catalunya, Jordi Girona 1-3, 08034 Barcelona, Catalonia, Spain.

Email address: jordi . castro@upc .edu (Jordi Castro)

Preprint submitted to Operations Research Letters 
$Q_{P}$ being a positive definite matrix and $\bar{x}$ the current point obtained by the interior-point algorithm. For instance, $Q_{P}$ was the identity matrix in [6]; and $Q_{P}$ was a diagonal matrix with small entries - dynamically updated at each interior-point iteration-in [1]. Unfortunately, these proximal point regularizations depend on the current point $\bar{x}$, and then they do not fit the general theory of structural optimization for interior-point methods [5]. In [3] the authors suggested the alternative regularized barrier problem

$$
B_{Q}(x, \mu) \triangleq c^{T} x+\mu\left(\frac{1}{2} x^{T} Q x-\sum_{i=1}^{n} \ln x_{i}-\sum_{i=1}^{n} \ln \left(u_{i}-x_{i}\right)\right)
$$

$Q$ being a diagonal positive semidefinite matrix. This regularized barrier function was shown to be a self-concordant barrier [3] for upper-bounded problems and thus it fits the general interiorpoint theory of [5]. It was shown in [3] than, due to this regularization term, the spectral properties of a preconditioned system were significantly improved. This allowed the efficient solution of the normal equations of some very large primal block-angular problems by means of a scheme that combines Cholesky factorizations and preconditioned conjugate gradients [2].

The KKT conditions for (2) are [7]:

$$
\begin{aligned}
A x & =b, \\
A^{T} y+z-w & =c, \\
X Z e & =\mu e, \\
(U-X) W e & =\mu e, \quad 0<x<u ;
\end{aligned}
$$

$e \in \mathbb{R}^{n}$ is a vector of 1's; $y \in \mathbb{R}^{m}, z, w \in \mathbb{R}^{n}$ are the Lagrange multipliers (or dual variables) of $A x=b, x \geq 0$ and $x \leq u$, respectively; and matrices $X, Z, U, W \in \mathbb{R}^{n \times n}$ are diagonal matrices made up of vectors $x, z, u, w$. The first two sets of inequalities of (5) impose, respectively, primal and dual feasibility; the last two impose complementarity. The solutions of system (5) for different $\mu$ values gives rise to an arc of strictly feasible primal-dual points known as the primaldual central path. As $\mu$ tends to 0 , the solutions of (5) converge to those of (1) and its dual. A primal-dual path-following algorithm attempts to follow the primal-dual central path. This is the algorithm implemented in packages like, e.g., CPLEX, XPress, MOSEK, etc.

The KKT conditions for (2) replacing $B(x, \mu)$ by the regularized version (4) are

$$
\begin{aligned}
A x & =b, \\
A^{T} y-\mu Q x+z-w & =c, \\
X Z e & =\mu e, \\
(U-X) W e & =\mu e, \quad 0<x<u .
\end{aligned}
$$

Note (5) and (6) only differ in the dual feasibility. System (6) will be referred as the the regularized KKT conditions, and the arc of primal-dual solutions for different $\mu$ values as the regularized primal-dual central path.

The purpose of this short paper is to show that the regularized primal-dual central path is well defined for (primal and dual) feasible problems: it exists and it is unique (i.e., for any $\mu$ there is a solution to (6), and this solution is unique); and it converges to a strictly complementary solution of (1). Section 2 shows the existence and uniqueness. Section 3 shows the convergence to a primal-dual solution with strict complementarity. We extend previous results $[4,7,8]$ for the standard central path defined by (5) to the new regularized version (6). 


\section{Existence and uniqueness of the regularized central path}

To simplify the notation, we will consider that the bounded problem (1) has been transformed to an equivalent problem without explicit upper bounds (i.e., adding slacks $s \in \mathbb{R}^{n}$, and constraints $x+s=u$ to $A x=b$, and including slacks in the vector of variables):

$$
\begin{array}{cl}
\min & c^{T} x \\
\text { s. to } & A x=b \\
& x \geq 0 .
\end{array}
$$

The dual of (7) is

$$
\begin{array}{cl}
\min & b^{T} y \\
\text { s. to } & A^{T} y+z=c \\
& z \geq 0 .
\end{array}
$$

The simplified primal and dual regularized logarithmic barrier problems are, respectively,

$$
\begin{aligned}
& \min c^{T} x+\mu\left(\frac{1}{2} x^{T} Q x-\sum_{i=1}^{n} \ln x_{i}\right) \\
& \text { s. to } \quad A x=b, \quad x>0
\end{aligned}
$$

and

$$
\begin{array}{ll}
\max & b^{T} y+\mu\left(-\frac{1}{2} x^{T} Q x+\sum_{i=1}^{n} \ln z_{i}\right) \\
\text { s. to } \quad A^{T} y-\mu Q x+z=c, \quad z>0 .
\end{array}
$$

The simplified regularized KKT conditions for either (9) or (10) are

$$
\begin{aligned}
A x & =b, \\
A^{T} y-\mu Q x+z & =c, \\
X Z e & =\mu e, \\
(x, z) & >0 .
\end{aligned}
$$

The primal-dual feasible set $\mathcal{F}$ and the strictly feasible set $\mathcal{F}^{0}$ are defined by

$$
\begin{aligned}
& \mathcal{F}=\left\{(x, y, z) \mid A x=b, A^{T} y-\mu Q x+z=c,(x, z) \geq 0\right\}, \\
& \mathcal{F}^{0}=\left\{(x, y, z) \mid A x=b, A^{T} y-\mu Q x+z=c,(x, z)>0\right\} .
\end{aligned}
$$

We start by proving the following preliminary Lemma, to be used later:

Lemma 1. If $\mathcal{F}^{0} \neq \oslash$ (i.e., the problem is strictly feasible) then for each $K \in \mathbb{R}, K \geq 0$, the set

$$
\left\{(x, z) \mid(x, y, z) \in \mathcal{F} \text { for some } y \text {, and } x^{T} z \leq K\right\}
$$

is bounded.

Proof. Let $(\bar{x}, \bar{y}, \bar{z})$ be any point in $\mathcal{F}^{0}$ and $(x, y, z)$ be any point in $\mathcal{F}$ such that $x^{T} z \leq K$. Since $A \bar{x}=b$ and $A x=b$ then $A(\bar{x}-x)=0$. Similarly, $A^{T}(\bar{y}-y)+(\bar{z}-z)-\mu Q(\bar{x}-x)=0$. Therefore,

$$
\begin{aligned}
(\bar{x}-x)^{T}(\bar{z}-z)= & (\bar{x}-x)^{T}\left(-A^{T}(\bar{y}-y)+\mu Q(\bar{x}-x)\right) \\
= & \mu(\bar{x}-x)^{T} Q(\bar{x}-x) \\
& -(\bar{x}-x)^{T} A^{T}(\bar{y}-y) \\
= & \mu(\bar{x}-x)^{T} Q(\bar{x}-x)-0 \cdot(\bar{y}-y) \\
= & \mu(\bar{x}-x)^{T} Q(\bar{x}-x),
\end{aligned}
$$


which can be recast as

$$
\bar{x}^{T} z+\bar{z}^{T} x=\bar{x}^{T} \bar{z}+x^{T} z-\mu(\bar{x}-x)^{T} Q(\bar{x}-x) .
$$

Since $x^{T} z \leq K$ and $\mu(\bar{x}-x)^{T} Q(\bar{x}-x) \geq 0$ because $Q$ is positive semidefinite,

$$
\bar{x}^{T} z+\bar{z}^{T} x \leq K+\bar{x}^{T} \bar{z}-\mu(\bar{x}-x)^{T} Q(\bar{x}-x) \leq K+\bar{x}^{T} \bar{z}
$$

The value $\xi=\min _{i=1, \ldots, n} \min \left(\bar{x}_{i}, \bar{z}_{i}\right)$ is positive, since $(\bar{x}, \bar{z})>0$. Then from (15) we have

$$
\xi e^{T}(x+z) \leq \bar{x}^{T} z+\bar{z}^{T} x \leq K+\bar{x}^{T} \bar{z}
$$

which means

$$
0 \leq x_{i} \leq \frac{1}{\xi}\left(K+\bar{x}^{T} \bar{z}\right), 0 \leq z_{i} \leq \frac{1}{\xi}\left(K+\bar{x}^{T} \bar{z}\right), i=1, \ldots, n,
$$

and hence (14) is bounded.

To show existence and uniqueness we first define the new set

$$
\mathcal{H}^{0}=\left\{(x, z) \mid(x, y, z) \in \mathcal{F}^{0} \text { for some } y\right\} .
$$

We also define the barrier function

$$
f_{\mu}(x, z)=\frac{1}{\mu} x^{T} z-\sum_{i=1}^{n} \ln \left(x_{i} z_{i}\right)
$$

with the following properties:

Lemma 2. 1. $f_{\mu}$ tends to $+\infty$ whenever $(x, z)$ approaches the boundary of $\mathcal{H}^{0}$, i.e., when any $x_{j}$ or $z_{j}$ approaches 0.

2. $f_{\mu}$ is strictly convex on $\mathcal{H}^{0}$.

3. $f_{\mu}$ is bounded below on $\mathcal{H}^{0}$.

4. Given $\mu>0$, and any $\kappa \in \mathbb{R}$, points $(x, z)$ of the level set $\mathcal{L}_{\kappa}=\left\{(x, z) \in \mathcal{H}^{0} \mid f_{\mu}(x, z) \leq \kappa\right\}$ satisfy

$$
x_{i} \in\left[M_{l}, M_{u}\right], z_{i} \in\left[M_{l}, M_{u}\right], \quad i=1, \ldots, n,
$$

for some positive numbers $M_{l}$ and $M_{u}$, and thus they are contained in compact subsets.

Proof. (We remark that the regularization term does not intervene in proofs of properties 1, 3 and 4 , and they are the same than for the standard central path; anyway, we recall them here for completeness).

Property 1 is straightforward.

For property 2, note that the second term $-\sum_{i=1}^{n} \ln \left(x_{i} z_{i}\right)$ is strictly convex (since its Hessian is positive definite). The first term is shown to be convex on $\mathcal{H}^{0}$. Indeed, if $\bar{x}$ is any point for which $A \bar{x}=b$, we have for any $(x, z) \in \mathcal{H}^{0}$ that $x^{T} z=x^{T}\left(c-A^{T} y+\mu Q x\right)=c^{T} x-\bar{x}^{T} A^{T} y+\mu x^{T} Q x=$ $c^{T} x-\bar{x}^{T}(c-z+\mu Q x)+\mu x^{T} Q x=c^{T} x-c^{T} \bar{x}+\bar{x}^{T} z-\mu \bar{x}^{T} Q x+\mu x^{T} Q x$, which is convex in $(x, z)$ since $Q \geq 0$. Hence, $f_{\mu}(x, z)$ is the sum of a convex and a strictly convex function, thus it is strictly convex. 
To show property 3 , we define $g(t)=t-\ln t-1$ and rewrite $f_{\mu}(x, z)$ as

$$
f_{\mu}(x, z)=\sum_{j=1}^{n} g\left(\frac{x_{j} z_{j}}{\mu}\right)+n-n \ln \mu .
$$

Function $g(t)$ is strictly convex in $(0, \infty), g(t) \geq 0$ for $t \in(0, \infty)$, and tends to $\infty$ when either $t \rightarrow 0$ or $t \rightarrow \infty$. Using $g(t) \geq 0$ in (18) we have

$$
f_{\mu}(x, z) \geq n-n \ln \mu=n(1-\ln \mu),
$$

i.e., $f_{\mu}(x, z)$ is bounded below.

Property 4 is shown by noting that by $(18) f_{\mu}(x, z) \leq \kappa$ if and only if

$$
\sum_{j=1}^{n} g\left(\frac{x_{j} z_{j}}{\mu}\right) \leq \bar{\kappa},
$$

where $\bar{\kappa}=\kappa-n+n \ln \mu$. Choosing a particular index $i=j$, and using that $g(t) \geq 0$, we have

$$
g\left(\frac{x_{i} z_{i}}{\mu}\right) \leq \bar{\kappa}-\sum_{j \neq i} g\left(\frac{x_{j} z_{j}}{\mu}\right) \leq \bar{\kappa} .
$$

Therefore, using that $g(t) \rightarrow \infty$ when either $t \rightarrow 0$ or $t \rightarrow \infty$, there exists a value $M$ such that

$$
\frac{1}{M} \leq x_{i} z_{i} \leq M, \quad i=1, \ldots, n
$$

Adding the terms in this expression we get

$$
x^{T} z=\sum_{i=1}^{n} x_{i} z_{i} \leq n M .
$$

By (20) and the boundedness established by Lemma 1 we know there exists a number $M_{u}$ such that $x_{i} \in\left(0, M_{u}\right]$ and $z_{i} \in\left(0, M_{u}\right]$ for all $i=1, \ldots, n$. Using (19), we have that $x_{i} \geq 1 /\left(M z_{i}\right) \geq$ $1 /\left(M M_{u}\right)$ for all $i$; for $z_{i}$ we obtain the same lower bound. (17) holds by setting $M_{l}=1 /\left(M M_{u}\right)$.

Finally, next Theorem 1 shows that for any $\mu>0$ the barrier function $f_{\mu}(x, z)$ defined by (16) reaches its minimum in $\mathcal{H}^{0}$, that the minimizer is unique, and that this means that the regularized KKT conditions (11) have a unique solution.

Theorem 1. If $F^{0} \neq \oslash$ and $\mu>0$, then $f_{\mu}(x, z)$ has a unique minimizer in $\mathcal{H}^{0}$, and (11) has a unique solution.

Proof. By property 4 of Lemma 2 we have that level sets $\mathcal{L}_{\kappa}=\left\{(x, z) \in \mathcal{H}^{0} \mid f_{\mu}(x, z) \leq \kappa\right\}$ of $f_{\mu}(x, z)$ are contained in a compact subset of $\mathcal{H}^{0}$, and thus $f_{\mu}(x, z)$ has a minimizer in $\mathcal{H}^{0}$. By property 2 of Lemma $2, f_{\mu}(x, z)$ is strictly convex, thus the minimizer will be unique.

We next show this unique minimizer corresponds to the unique solution of (11). This minimizer solves the linearly constrained minimization problem

$$
\min f_{\mu}(x, z) \text { s. to } A x=b, A_{5}^{T} y+z-\mu Q x=c,(x, z)>0 .
$$


From the Lagrangian

$$
\begin{aligned}
\mathcal{L}(x, y, z, v, w)= & f_{\mu}(x, z)+v^{T}(A x-b) \\
& +w^{T}\left(A^{T} y+z-\mu Q x-c\right)
\end{aligned}
$$

we obtain the KKT conditions of (21)

$$
\begin{aligned}
\frac{d \mathcal{L}}{d x} & =\frac{d f_{\mu}}{d x}+A^{T} v-\mu Q w \\
& =\frac{1}{\mu} Z e-X^{-1} e+A^{T} v-\mu Q w=0, \\
\frac{d \mathcal{L}}{d y} & =A w=0, \\
\frac{d \mathcal{L}}{d z} & =\frac{d f_{\mu}}{d z}+w=\frac{1}{\mu} X e-Z^{-1} e+w=0 .
\end{aligned}
$$

By combining the first and third equalities of (22) we obtain

$$
A^{T} v=X^{-1} e-\frac{1}{\mu} Z e+\mu Q\left(Z^{-1} e-\frac{1}{\mu} X e\right) .
$$

By combining the second and third we find that

$$
A\left(Z^{-1} e-\frac{1}{\mu} X e\right)=0
$$

which means

$$
\left(Z^{-1} e-\frac{1}{\mu} X e\right)^{T} A^{T} v=0 .
$$

Using the above result in (23) we have

$$
\left(Z^{-1} e-\frac{1}{\mu} X e\right)^{T}\left(X^{-1} e-\frac{1}{\mu} Z e+\mu Q\left(Z^{-1} e-\frac{1}{\mu} X e\right)\right)=0,
$$

or equivalently,

$$
\begin{gathered}
\left(Z^{-1} e-\frac{1}{\mu} X e\right)^{T}\left(X^{-1} e-\frac{1}{\mu} Z e\right) \\
+\quad\left(Z^{-1} e-\frac{1}{\mu} X e\right)^{T} \mu Q\left(Z^{-1} e-\frac{1}{\mu} X e\right)=0 .
\end{gathered}
$$

The first term of (24) can be written as

$$
\begin{aligned}
\left(Z^{-1} e-\frac{1}{\mu} X e\right)^{T}\left(X^{\frac{-1}{2}} Z^{\frac{1}{2}}\right)\left(X^{\frac{1}{2}} Z^{\frac{-1}{2}}\right)\left(X^{-1} e-\frac{1}{\mu} Z e\right) & = \\
\left(X^{\frac{-1}{2}} Z^{\frac{-1}{2}} e-\frac{1}{\mu} X^{\frac{1}{2}} Z^{\frac{1}{2}} e\right)^{T}\left(X^{\frac{-1}{2}} Z^{\frac{-1}{2}} e-\frac{1}{\mu} X^{\frac{1}{2}} Z^{\frac{1}{2}} e\right) & = \\
\left\|(X Z)^{-1 / 2} e-\frac{1}{\mu}(X Z)^{1 / 2} e\right\|_{2}^{2} & \geq 0,
\end{aligned}
$$

\|\|$_{2}$ being the Euclidean norm. Since $Q \geq 0$ and $\mu>0$, the second term of (24) is greater or equal than zero. Therefore (24) holds if and only if

$$
\begin{array}{rl}
\left\|(X Z)^{-1 / 2} e-\frac{1}{\mu}(X Z)^{1 / 2} e\right\|_{2}^{2} & =0 \\
\left(Z^{-1} e-\frac{1}{\mu} X e\right)^{T} \mu Q\left(Z^{-1} e-\frac{1}{\mu} X e\right) & =0 . \\
6 & 0
\end{array}
$$


From (25) we have $(X Z)^{-1 / 2} e=\frac{1}{\mu}(X Z)^{1 / 2} e$, and therefore $X Z e=\mu e . X Z e=\mu e$ if and only if $Z^{-1} e-\frac{1}{\mu} X e=0$, and then (26) holds. Therefore, the unique minimizer of (21) satisfies not only the feasibility conditions of (11), but also the $\mu$-complementarity condition, and the proof is complete.

\section{Convergence of the regularized central path}

Two properties are first proved. The first one shows the regularized central path is bounded.

Proposition 1. Let $(x(\mu), y(\mu), z(\mu))$ be on the regularized central path defined by (11). Then $(x(\mu), z(\mu))$ is bounded for all $0<\mu<\bar{\mu}$ and any given $0<\bar{\mu}<\infty$.

Proof. Since $(x(\mu), y(\mu), z(\mu))$ solves $(11), x^{T}(\mu) z(\mu)=n \mu$. Therefore, using Lemma $1,(x(\mu), z(\mu))$ is bounded.

The second property shows the evolution of the objectives $c^{T} x$ and $b^{T} y$, and the regularized barriers $P_{Q}(x) \triangleq \frac{1}{2} x^{T} Q x-\sum_{i=1}^{n} \ln x_{i}$ and $D_{Q}(x, z) \triangleq-\frac{1}{2} x^{T} Q x+\sum_{i=1}^{n} \ln z_{i}$ of, respectively, (9) and (10), along the regularized central path.

Proposition 2. Let $(x(\mu), y(\mu), z(\mu))$ be on the regularized central path defined by (11). Therefore, for any $0<\mu_{2}<\mu_{1}$

$$
\begin{array}{ll}
c^{T} x\left(\mu_{2}\right)<c^{T} x\left(\mu_{1}\right), & P_{Q}\left(x\left(\mu_{2}\right)\right)>P_{Q}\left(x\left(\mu_{1}\right)\right), \\
b^{T} y\left(\mu_{2}\right)>b^{T} y\left(\mu_{1}\right), & D_{Q}\left(x\left(\mu_{2}\right)\right)<D_{Q}\left(x\left(\mu_{1}\right)\right) .
\end{array}
$$

Proof. For notational simplicity, let us denote by $\left(x^{i}, y^{i}, s^{i}\right)$ the point $\left(x\left(\mu_{i}\right), y\left(\mu_{i}\right), z\left(\mu_{i}\right)\right)$ on the regularized central path. Since $x^{1}$ and $x^{2}$ solve (9) for, respectively, $\mu_{1}$ and $\mu_{2}$, and the objective function of (9) is strictly convex, we have

$$
c^{T} x^{1}+\mu_{1} P_{Q}\left(x^{1}\right)<c^{T} x^{2}+\mu_{1} P_{Q}\left(x^{2}\right)
$$

and

$$
c^{T} x^{2}+\mu_{2} P_{Q}\left(x^{2}\right)<c^{T} x^{1}+\mu_{2} P_{Q}\left(x^{1}\right) .
$$

Adding (27) and (28), we obtain

$$
\left(\mu_{2}-\mu_{1}\right)\left(P_{Q}\left(x^{1}\right)-P_{Q}\left(x^{2}\right)\right)>0 .
$$

Since $\mu_{2}<\mu_{1}$, from (29) we have

$$
P_{Q}\left(x^{2}\right)>P_{Q}\left(x^{1}\right) .
$$

Using (30) in (28), we see that $c^{T} x^{2}<c^{T} x^{1}$.

Similarly, since $\left(x^{1}, y^{1}, z^{1}\right)$ and $\left(x^{2}, y^{2}, z^{2}\right)$ solve (10) for, respectively, $\mu_{1}$ and $\mu_{2}$, and the objective of (10) is strictly concave on the feasible region (i.e., the reduced Hessian-pre and post multiplied by the Jacobian of constraints-is negative definite), we have

$$
b^{T} y^{1}+\mu_{1} D_{Q}\left(x^{1}, z^{1}\right)>b^{T} y^{2}+\mu_{1} D_{Q}\left(x^{2}, z^{2}\right)
$$

and

$$
b^{T} y^{2}+\mu_{2} D_{Q}\left(x^{2}, z^{2}\right)>b^{T} y^{1}+\mu_{2} D_{Q}\left(x^{1}, z^{1}\right) .
$$

Adding (31) and (32), and using that $\mu_{2}<\mu_{1}$, we conclude that $D_{Q}\left(x^{2}\right)<D_{Q}\left(x^{1}\right)$. Using this result in (32), we finally obtain that $b^{T} y^{2}>b^{T} y^{1}$ 
Next proposition shows the regularized central path converges to an optimal solution.

Proposition 3. The regularized central path sequence $(x(\mu), y(\mu), z(\mu))$ converges to an optimal solution of (7) and (8) as $\mu \rightarrow 0$.

Proof. By Proposition $1,(x(\mu), z(\mu))$ is bounded and then it has a limit point $(x(0), z(0))$ as $\mu \rightarrow 0$. Any $(x(\mu), z(\mu))$ in the subsequence whose limit is $(x(0), z(0))$ satisfies (11), i.e., $A x(\mu)=b$, $c-z(\mu)+\mu Q x(\mu) \in \operatorname{range}\left(A^{T}\right), x(\mu)^{T} z(\mu)=n \mu,(x(\mu), z(\mu)) \geq 0$. Since range $\left(A^{T}\right)$ is a closed set, as $\mu \rightarrow 0$ there is a $y(0)$ such that $A x(0)=b, c-z(0)=A^{T} y(0), x(0)^{T} z(0)=0,(x(0), z(0)) \geq 0$, and thus $(x(0), y(0), z(0))$ is an optimal solution of (7) and (8).

A significant difference between the standard central path defined by (5) and the regularized central defined by (6) is that the regularized central path is not guaranteed to converge to the analytic center of the optimal set for any regularization matrix $Q$. This can be illustrated by the following small example. Consider the maximization of $x_{1}$ over the unit square $[0,1]^{2}$ defined by constraints $0 \leq x_{1} \leq 1$, and $0 \leq x_{2} \leq 1$. This problem is formulated in standard form as

$$
\begin{array}{cl}
\min & -x_{1} \\
\text { s. to } & x_{1}+x_{3}=1 \\
& x_{2}+x_{4}=1 \\
& x_{i} \geq 0 \quad i=1, \ldots, 4
\end{array}
$$

Considering a diagonal regularization matrix $Q=\operatorname{diag}\left(q_{1}, q_{2}, q_{3}, q_{4}\right), q_{i} \geq 0, i=1, \ldots, 4$, conditions (11) for this problem are

$$
\begin{aligned}
x_{1}+x_{3} & =1 \\
x_{2}+x_{4} & =1 \\
y_{1}-\mu q_{1} x_{1}+z_{1} & =-1 \\
y_{2}-\mu q_{2} x_{2}+z_{2} & =0 \\
y_{1}-\mu q_{3} x_{3}+z_{3} & =0 \\
y_{2}-\mu q_{4} x_{4}+z_{4} & =0 \\
x_{i} z_{i} & =\mu i=1, \ldots, 4 \\
\left(x_{i}, z_{i}\right) & >0 \quad i=1, \ldots, 4 .
\end{aligned}
$$

If $Q=0$, i.e., there is no regularization, the solution of (34) for $x_{1}$ and $x_{2}$ is shown to be

$$
x_{1}=\frac{1-2 \mu+\sqrt{4 \mu^{2}+1}}{2}, \quad x_{2}=\frac{1}{2} .
$$

As $\mu \rightarrow \infty$ the central path point tends to $\left(x_{1}, x_{2}\right) \rightarrow(1 / 2,1 / 2)$, the analytic center of the feasible set. As $\mu \rightarrow 0$, the optimal solution $\left(x_{1}^{*}, x_{2}^{*}\right) \rightarrow(1,1 / 2)$ is obtained, which coincides with the analytic center of the optimal face (the segment $\overline{(1,0)(1,1)}$ ).

If $Q \neq 0$, the solution of (34) for $x_{1}$ and $x_{2}$ is provided by the cubic equations

$$
\begin{aligned}
\mu\left(q_{1}+q_{3}\right) x_{1}^{3}-\left(2 \mu q_{3}+\mu q_{1}+1\right) x_{1}^{2}+\left(\mu q_{3}-2 \mu+1\right) x_{1}+\mu & =0 \\
\left(q_{2}+q_{4}\right) x_{2}^{3}-\left(2 q_{4}+q_{2}\right) x_{2}^{2}+\left(q_{4}-2\right) x_{2}+1 & =0 .
\end{aligned}
$$

Note that $x_{2}$ does not depend on $\mu$, and for some $Q, x_{2}$ may be different than $1 / 2$, i.e., the optimal point does not converge to the analytic center of the optimal face. For instance, for the particular values $q_{i}=10 i, i=1, \ldots, 4$ we obtain $x_{2}=0.64548$. As $\mu \rightarrow \infty, x_{1} \rightarrow 0.70178$, and as 


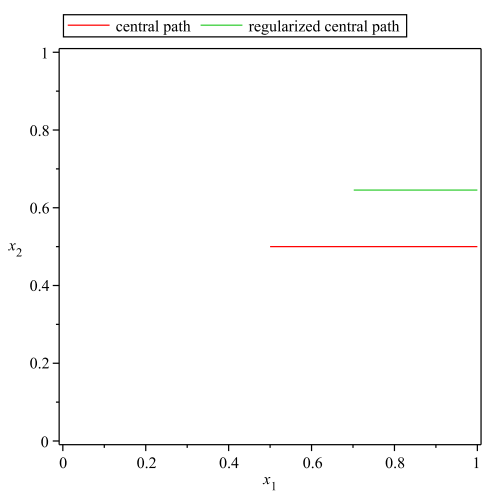

Figure 1: Central path and regularized central for problem (33) on $\left(x_{1}, x_{2}\right)$ space.

$\mu \rightarrow 0, x_{1} \rightarrow 1$. The other two solutions of each cubic equation are discarded since they do not satisfy some equations of (34). Figure 1 shows the central path and the regularized central path, for the previous particular $Q$, on the $\left(x_{1}, x_{2}\right)$ space. Note, however, that for some $Q$ the regularized central path, not only converges to the analytic center of the optimal solution set, but also coincides with the central path. For instance, in this example, this happens if $q_{1}=q_{2}=q_{3}=$ $q_{4}$.

It is also worth to mention that for problems with a unique optimal point, both the regularized and standard central path converge to this point, but, depending on $Q$, with different trajectories. However, in practice, since small regularizations are used, these trajectories are similar. To illustrate this situation, consider the simple problem

$$
\begin{array}{cl}
\min & x_{1}+x_{2} \\
\text { s. to } & x_{1} \geq 0, x_{2} \geq 0,
\end{array}
$$

with a unique optimal solution. Conditions (11) for this problem are

$$
\begin{array}{rcr}
-\mu q_{i} x_{i}+z_{i} & =1 & i=1,2 \\
x_{i} z_{i} & =\mu & i=1,2 \\
\left(x_{i}, z_{i}\right) & >0 & i=1,2 .
\end{array}
$$

If $Q=0$, the central path is $\left(x_{1}=\mu, x_{2}=\mu, z_{1}=1, z_{2}=1\right)$. If $Q \neq 0$, the regularized central path is

$$
\begin{array}{ll}
x_{i}=\frac{-1+\sqrt{1+4 \mu^{2} q_{i}}}{2 \mu q_{i}} & i=1,2 \\
z_{i}=\frac{2 \mu^{2} q_{i}}{-1+\sqrt{1+4 \mu^{2} q_{i}}} & i=1,2 .
\end{array}
$$

As $\mu \rightarrow 0$, then $x_{i} \rightarrow 0$ and $z_{i} \rightarrow 1, i=1,2$, thus the regularized central path provides the optimal solution. However, the trajectories of the central path and regularized central path are different, as shown in Figure 2 for $q_{1}=1$ and $q_{2}=25$. Note that as $\mu \rightarrow \infty,\left(x_{1}, x_{2}\right) \rightarrow(\infty, \infty)$ in the central path, whereas $\left(x_{1}, x_{2}\right) \rightarrow(1,1 / 5)$ in the regularized central path

The previous discussion can be summarized in the following result. 


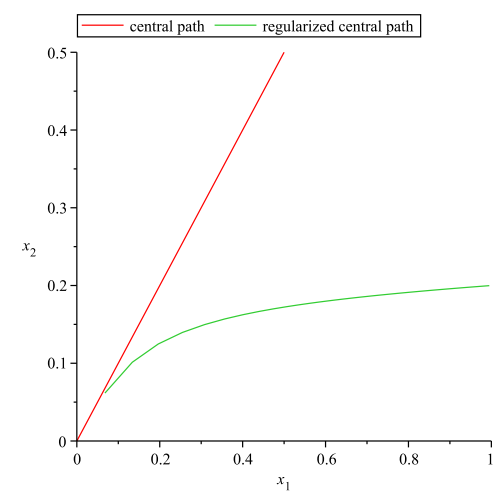

Figure 2: Central path (only part between $\mu=0.5$ and $\mu=0$ ) and regularized central path for problem (35) on $\left(x_{1}, x_{2}\right)$ space.

Proposition 4. The regularized central path sequence $(x(\mu), y(\mu), z(\mu))$ is not guaranteed to converge to the analytic center of the optimal set as $\mu \rightarrow 0$ (unlike the standard central path).

Proof. The above problem (33) is a counterexample that proves this proposition.

Finally, the below result shows that, like the central path, the regularized central path converges to a strictly complementary primal-dual solution.

Theorem 2. The regularized central path sequence $(x(\mu), y(\mu), z(\mu))$ converges to a strictly complementary primal-dual solution as $\mu \rightarrow 0$.

Proof. By the Goldman-Tucker theorem, there exists a strictly complementary solution $\left(x^{*}, y^{*}, z^{*}\right)$, i.e., $x^{*}$ solves $(7),\left(y^{*}, z^{*}\right)$ solves $(8)$, and $x_{\mathcal{B}}>0, x_{\mathcal{N}}=0, z_{\mathcal{B}}=0, z_{\mathcal{N}}>0$, where

$$
\begin{aligned}
\mathcal{B} & =\left\{i \in\{1, \ldots, n\}: x_{i}^{*}>0\right\}, \\
\mathcal{N} & =\left\{i \in\{1, \ldots, n\}: z_{i}^{*}>0\right\} .
\end{aligned}
$$

are the sets of the complementarity partition (i.e., $\mathcal{B} \cup \mathcal{N}=\{1, \ldots, n\}$, and $\mathcal{B} \cap \mathcal{N}=\emptyset$ ). Let $(x(\mu), y(\mu), z(\mu))$ be a point of the regularized central path for some $\mu$, i.e., this point solves (11). Since the complementary solution $\left(x^{*}, y^{*}, z^{*}\right)$ solves the KKT conditions of (7) and (8), namely, $A x^{*}=b, A^{T} y^{*}+z^{*}=c,\left(x^{*}\right)^{T} z^{*}=0,\left(x^{*}, z^{*}\right) \geq 0$, we have $A\left(x^{*}-x(\mu)\right)=0$ and $z^{*}-z(\mu)=-A^{T}\left(y^{*}-y(\mu)\right)-\mu Q x(\mu)$. Therefore

$$
\left(x^{*}-x(\mu)\right)^{T}\left(z^{*}-z(\mu)\right)=-\mu\left(x^{*}-x(\mu)\right)^{T} Q x(\mu) .
$$

Since $\left(x^{*}-x(\mu)\right)^{T}\left(z^{*}-z(\mu)\right)$ also satisfies

$$
\left(x^{*}-x(\mu)\right)^{T}\left(z^{*}-z(\mu)\right)=n \mu-\left(x^{*}\right)^{T} z(\mu)-\left(z^{*}\right)^{T} x(\mu),
$$

combining (37) and (38) we have

$$
0 \leq\left(x^{*}\right)^{T} z(\mu)+\left(z^{*}\right)^{T} x(\mu)=\mu\left(n+\left(x^{*}-x(\mu)\right)^{T} Q x(\mu)\right) .
$$


Since $x^{*}$ is bounded, and by Proposition $1 x(\mu)$ is also bounded for all $0<\mu<\bar{\mu}$ and any given $0<\bar{\mu}<\infty$, there exists a bounded value in $\mathbb{R}, 0 \leq C<\infty$ such that

$$
\begin{aligned}
C \geq & \left\{\left(x^{*}-x(\mu)\right)^{T} Q x(\mu): x^{*}\right. \text { solves (7), } \\
& x(\mu) \text { solves }(11), 0<\mu<\bar{\mu}\},
\end{aligned}
$$

for any given $0<\bar{\mu}<\infty$. Then

$$
0 \leq \sum_{i \in \mathcal{B}} x_{i}^{*} z_{i}(\mu)+\sum_{i \in \mathcal{N}} z_{i}^{*} x_{i}(\mu) \leq \mu(n+C),
$$

where $n+C \geq n$. Since $x_{i}(\mu) z_{i}(\mu)=\mu, i=1, \ldots, n,(40)$ can be rewritten as

$$
0 \leq \sum_{i \in \mathcal{B}} \frac{x_{i}^{*}}{x_{i}(\mu)}+\sum_{i \in \mathcal{N}} \frac{z_{i}^{*}}{z_{i}(\mu)} \leq(n+C) .
$$

Thus we have

$$
x_{i}(\mu) \geq \frac{x_{i}^{*}}{n+C}>0 \quad i \in \mathcal{B}, \quad z_{i}(\mu) \geq \frac{z_{i}^{*}}{n+C}>0 \quad i \in \mathcal{N} .
$$

Therefore, as $\mu \rightarrow 0, x_{\mathcal{B}}(0)>0$ and $z_{\mathcal{N}}(0)>0$, i.e., the regularized central path converges to a strictly complementary primal-dual solution.

As a corollary of Theorem 2, we have that under some conditions, the regularized central path is guaranteed to converge to the analytic center of the optimal face, like the standard central path.

Corollary 1. Let $\left(x^{*}, y^{*}, z^{*}\right)$ be the analytic center of the primal and dual optimal set. If $C=0$, $C$ defined in (39), then the regularized central path sequence $(x(\mu), y(\mu), z(\mu))$ converges to the analytic center of the optimal set $\left(x^{*}, y^{*}, z^{*}\right)$.

Proof. The analytic center of the optimal set $\left(x^{*}, y^{*}, z^{*}\right)$ is a strictly complementary solution. Then, by (41), and using that $C=0$, we have

$$
\frac{\sum_{i \in \mathcal{B}} \frac{x_{i}^{*}}{x_{i}(\mu)}+\sum_{i \in \mathcal{N}} \frac{z_{i}^{*}}{z_{i}(\mu)}}{n} \leq 1 .
$$

Therefore, by the inequality of arithmetic and geometric means of positive numbers,

$$
\begin{gathered}
\left(\prod_{i \in \mathcal{B}} \frac{x_{i}^{*}}{x_{i}(\mu)}\right)\left(\prod_{i \in \mathcal{N}} \frac{z_{i}^{*}}{z_{i}(\mu)}\right) \leq 1, \\
\left(\prod_{i \in \mathcal{B}} x_{i}^{*}\right)\left(\prod_{i \in \mathcal{N}} z_{i}^{*}\right) \leq\left(\prod_{i \in \mathcal{B}} x_{i}(\mu)\right)\left(\prod_{i \in \mathcal{N}} z_{i}(\mu)\right) .
\end{gathered}
$$

Of all the points on the optimal face, the analytic center is the unique maximizer of $\left(\prod_{i \in \mathcal{B}} x_{i}\right)\left(\prod_{i \in \mathcal{N}} z_{i}\right)$, and then, as $\mu \rightarrow 0$, we must have

$$
\left(\prod_{i \in \mathcal{B}} x_{i}^{*}\right)\left(\prod_{i \in \mathcal{N}} z_{i}^{*}\right)=\left(\prod_{i \in \mathcal{B}} x_{i}(0)\right)\left(\prod_{i \in \mathcal{N}} z_{i}(0)\right),
$$

i.e., $x_{\mathcal{B}}(0)=x_{\mathcal{B}}^{*}$ and $z_{\mathcal{N}}(0)=z_{\mathcal{N}}^{*}$. 
For instance, note that the conditions of Corollary 1 hold in example (35): $x^{*}=0$ is the unique solution - thus the analytic center — of the optimal set, and $C=0 \geq-x(\mu)^{T} Q x(\mu)$, since $Q \geq 0$.

\section{Acknowledgments}

The authors thank an anonymous referee whose comments greatly improved the paper (in particular they suggested Section 3). This work has been supported by grants MEC MTM200605550, MICINN MTM2009-08747 and SGR-2009-1122.

\section{References}

[1] A. Altman, J. Gondzio, Regularized symmetric indefinite systems in interior point methods for linear and quadratic optimization, Optimization Methods and Software 11 (1999) 275-302.

[2] J. Castro, A specialized interior-point algorithm for multicommodity network flows, SIAM Journal on Optimization 10 (2000) 852-877.

[3] J. Castro, J. Cuesta, Quadratic regularizations in an interior-point method for primal block-angular problems, Mathematical Programming, DOI:10.1007/s10107-010-0341-2, in press (2010).

[4] L.M. Graña Drummond, B.F. Svaiter, On well definedness of the central path, Journal of Optimization Theory and Applications 102 (1999) 223-237

[5] Y. Nesterov, Introductory Lectures on Convex Optimization: A Basic Course, Kluwer, 2004.

[6] R. Setiono, Interior proximal point algorithm for linear programs, Journal of Optimization Theory and Applications 74 (1992) 425-444.

[7] S.J. Wright, Primal-Dual Interior-Point Methods, SIAM, 1996.

[8] Y. Ye, Interior Point Algoriths. Theory and Analysis, Wiley, 1997. 